\title{
DOSE DE INOCULANTE TURFOSO PARA SOJA EM PLANTIO DIRETO ${ }^{1}$
}

\author{
DOSE OF PEAT INOCULANT FOR SOYBEAN UNDER NO-TILLAGE
}

\author{
Ben-Hur Costa de Campos ${ }^{2}$
}

\section{RESUMO}

Embora a inoculação na cultura da soja seja uma prática amplamente difundida, ainda não há um consenso sobre a quantidade de inoculante turfoso a utilizar-se em plantio direto. Enquanto no Paraná a quantidade recomendada é de $500 \mathrm{~g}$ de inoculante para $50 \mathrm{~kg}$ de sementes, no Rio Grande do Sul e Santa Catarina é de 200g, o que evidencia a necessidade de se intensificar as pesquisas nessa área. $O$ trabalho foi realizado, na safra 1994/95, em área conduzida há cinco anos no sistema plantio direto, em Cruz Alta, RS, com o objetivo de avaliar o efeito da dose de inoculante turfoso na soja em plantio direto. Os tratamentos foram: testemunha sem inoculação, doses de 250, 500, 750 e $1000 \mathrm{~g}$ de inoculante por $50 \mathrm{~kg}$ de semente e um tratamento com $200 \mathrm{~kg}$ de $\mathrm{N} / \mathrm{ha}$, parcelados, sem inoculação. Não houve diferenças entre a testemunha sem inoculação e as doses de inoculante para número e peso de nódulos, massa seca da parte aérea, massa seca de raízes, altura de planta e teor de $N$ na folha. $O$ tratamento com $N$ mineral apresentou menor número e peso de nódulos e maior produção de matéria seca da parte aérea, porém, neste último parâmetro, não diferiu da testemunha sem inoculação, 250 e $500 \mathrm{~g}$ de inoculante. Com a aplicação de $\mathrm{N}$ mineral, a altura de planta foi significativamente superior à testemunha e às doses de inoculante. Não houve efeito positivo da inoculação sobre o rendimento de grãos, já que este não diferiu significativamente entre as doses de inoculante e a testemunha sem inoculação. Estes resultados indicam não haver justificativa para o uso de inoculante em áreas a partir de cinco anos de plantio direto.

Palavras-chave: fixação biológica de $\mathrm{N}_{2}$, inoculação, Glycine max, Bradyrhizobium japonicum.

\section{SUMMARY}

Inoculation in the soybean crop is a practice widely adopted all over the world. Despite of this fact, so far researchers have not come to a agreement regarding the amount of peat inoculant to be used under the no-tillage cultivation system. While in Paraná State the recommended amount is of $500 \mathrm{~g}$ of inoculant for $50 \mathrm{~kg}$ of seeds, in Rio Grande do Sul and Santa Catarina States this recommendation is of $200 \mathrm{~g}$, what brings into evidence the need to intensify research in this particular area. This study was carried out in the 1994/95 cropping season, in an area cultivated under the no-tillage system for five years, in Cruz Alta,
Rio Grande do Sul State, Brazil, aiming at evaluating the effect of the peat inoculant dose on soybean under no-tillage. The treatments used were: check without inoculation, inoculant dosis of 250, 500, 750 and $1000 \mathrm{~g}$ for $50 \mathrm{~kg}$ of seeds and a treatment with $200 \mathrm{~kg}$ of N/ha, applied in parcels, without inoculation. There was no difference between the check plots without inoculation and inoculant dosis for number and weight of nodules, dry matter of the plant shoots, dry matter of the roots, plant height and $N$ content in the leaf. The treatment with mineral nitrogen showed a smaller number and weight of nodules and higher production of dry matter of the plant shoots, but this last parameter did not differ from the treatments: check without inoculation, 250 and $500 \mathrm{~g}$ of inoculant. With mineral $N$ application, plant height was significantly higher than the check and the inoculant dosis treatments. There was no positive effect of the inoculant on grain yield. These results led us to conclude that there is no need to use inoculant in areas after five years under the no-tillage cultivation system.

Key words: nitrogen biological fixation, inoculation, Glycine max, Bradyrhizobium japonicum.

\section{INTRODUÇÃO}

A soja [Glycine $\max (\mathrm{L}$.$) Merrill] é con-$ siderada a principal cultura comercial no Sul do país, com grande expressão na região do planalto do Rio Grande do Sul.

No desenvolvimento da cultura, um importante componente químico é o nitrogênio, que pode ser obtido do solo, a partir da decomposição da matéria orgânica, de fertilizantes nitrogenados e da associação simbiótica com bactérias da espécie Bradyrhizobium japonicum, através da fixação do nitrogênio atmosférico. Do ponto de vista econômico e ecológico, este último processo é considerado o mais importante, sendo que atualmente não são mais recomendados fertilizantes nitrogenados para a cultura da soja, pois a inoculação das sementes, a cada cultivo, supre as necessidades de nitrogênio da

\footnotetext{
${ }^{1}$ Trabalho apresentado na XXIII Reunião de Pesquisa de Soja da Região Sul, Porto Alegre (RS), em 01-03 de agosto de 1995.

${ }^{2}$ Engenheiro Agrônomo, Mestre, Pesquisador da Fundação Centro de Experimentação e Pesquisa Fecotrigo (FUNDACEP FECOTRIGO), RS 342, km 14, CP 10, 98100-970, Cruz Alta, RS. E-mail: fundacep@azcomnet.com.br.
} 
planta (REUNIÃO..., 1993). Segundo VARGAS et al. (1982), a soja é uma leguminosa que possui um alto nível de fixação de $\mathrm{N}_{2}$, quando bem nodulada, sendo capaz de apresentar produções de até 4 t.ha $^{-1}$ sem o uso de fertilizantes nitrogenados.

Os rizóbios presentes nos solos onde se cultivam leguminosas podem não trazer benefícios à cultura, o que levou à identificação e seleção, em laboratório, de estirpes mais eficientes a serem utilizadas como inoculantes. A introdução desses organismos, entretanto, só será bem sucedida se as estirpes selecionadas forem capazes de competir com as nativas do solo (OLIVEIRA \& VIDOR, 1984b).

Os inoculantes devem ser fabricados com estirpes recomendadas pelos laboratórios de pesquisa governamentais e possuir um padrão mínimo de 10 milhões de células de rizóbio por grama do veículo (FREIRE, 1992). Dessa forma, não somente o número inicial de células da bactéria é importante, mas principalmente o número que entra em contato com a semente durante a germinação. Nesse processo, a bactéria necessita de condições adequadas para a sua sobrevivência, desde a saída da fábrica até o momento da inoculação. Sabe-se que muitas vezes, essas condições não são atendidas, reduzindo o número de bactérias no inoculante.

Nos últimos anos, tem-se dado ênfase ao cultivo da soja no sistema plantio direto, pelas vantagens deste sistema quando comparado ao sistema convencional, com destaque à maior conservação do solo (BERTONI \& LOMBARDI NETO, 1990). O plantio direto tem a característica de ser um sistema de manejo no qual é evitada a mobilização do solo, criando um novo ambiente ecológico, diferente do sistema convencional, em conseqüência de um aumento de matéria orgânica (SÁ, 1993; CAMPOS et al., 1995), de nutrientes (ELTZ et al., 1989; SÁ, 1993) e da atividade microbiana (CAMPOS $\boldsymbol{e t}$ al., 1995) nos primeiros centímetros do solo. Além disso, provoca alterações nas condições de temperatura e umidade, propiciadas pela cobertura vegetal (BRAGAGNOLO \& MIELNICZUK, 1990). Assim, torna-se importante a avaliação de estirpes de rizóbio que apresentem bom desempenho na fixação de $\mathrm{N}_{2}$ nestas condições de manejo, pois as bactérias deste grupo foram selecionadas e avaliadas, por várias décadas, no sistema convencional.

Há dúvidas sobre a prática da inoculação na cultura da soja, em condições de plantio direto. Entre elas, destaca-se a necessidade de inoculação durante todos os cultivos, em áreas já cultivadas com essa leguminosa e a eficácia dos inoculantes usados, principalmente nos últimos anos, com a introdução de novas estirpes e aumento da área de plantio direto.
Outra questão que merece ser melhor estudada se refere à quantidade de inoculante a ser usada, pois a recomendação para o Paraná é de $500 \mathrm{~g}$ de inoculante para $50 \mathrm{~kg}$ de sementes (EMBRAPACNPSo/OCEPAR, 1993), enquanto que, para o Rio Grande do Sul e Santa Catarina, é de $200 \mathrm{~g}$ (REUNIÃO..., 1993).

O presente trabalho teve por objetivo avaliar o efeito da quantidade de inoculante na cultura da soja, em plantio direto.

\section{MATERIAL E MÉTODOS}

$\mathrm{O}$ experimento foi desenvolvido em área experimental localizada na FUNDACEP, Cruz Alta, RS, na safra 1994/95. O solo ocorrente é classificado como latossolo vermelho-escuro, pertencente à unidade de mapeamento Passo Fundo, fase argilosa, com as seguintes características na profundidade de 0 a 20cm: argila $490 \mathrm{~g} \mathrm{~kg}^{-1}$; $\mathrm{pH}$ (água) 6,1; índice SMP 6,6; P 3,2mg I-1 $\mathrm{K}^{-1} 1 \mathrm{mg} \mathrm{I}^{-1}$; matéria orgânica $28 \mathrm{~g} \mathrm{~kg}^{-1}$; Al $0,0 \mathrm{cmol}_{\mathrm{c}} I^{-1} ; \mathrm{Ca} 6,9 \mathrm{cmol}_{\mathrm{c}} I^{-1} ; \mathrm{Mg}$ $4,0 \mathrm{cmol}_{\mathrm{c}} I^{-1}$; CTC 12,0 $\mathrm{cmol}_{\mathrm{c}} I^{-1} ; \mathrm{H}+\mathrm{Al} 0,9 \mathrm{cmol}_{\mathrm{c}} I^{-1}$. Esta área apresentava-se no quinto ano de plantio direto, após ter sido cultivada por vários anos no sistema convencional, com culturas como soja, milho, trigo, aveia, entre outras. Nestes anos anteriores, a soja, a principal cultura de verão, sempre foi inoculada.

O delineamento foi de blocos ao acaso, com quatro repetições. Os tratamentos foram os seguintes: testemunha sem inoculação e doses de $250,500,750$ e $1000 \mathrm{~g}$ de inoculante por $50 \mathrm{~kg}$ de semente e $200 \mathrm{~kg}$ de $\mathrm{N}$ mineral/ha, parcelados, sem inoculação. Foi utilizado inoculante turfoso comercializado na região, contendo as estirpes Semia 587 + Semia 5019. Este inoculante foi adquirido diretamente da indústria e armazenado numa sala de temperatura amena, até o momento de seu uso. Como adubo mineral foi utilizado a uréia.

Cada parcela teve $3,0 \times 8,0 \mathrm{~m}$, com oito linhas de soja. Foram deixadas ruas de $1,5 \mathrm{~m}$ entre as parcelas, para evitar a contaminação entre os tratamentos e de 8,0m entre os blocos, para facilitar o tráfego de máquinas. Em cada parcela, a área útil foi composta pelas 6 linhas centrais de soja, desprezando-se $1,0 \mathrm{~m}$ em cada extremidade.

A adubação foi feita de acordo com a análise de solo, seguindo a recomendação para o sistema plantio direto. Esta foi realizada com $250 \mathrm{~kg}$ $\mathrm{ha}^{-1}$ da mistura de fosfato natural + cloreto de potássio correspondendo a fórmula 0-21-21.

Foi usada uma máquina betoneira para o tratamento das sementes com o fungicida Thiabendazole na dosagem de $10 \mathrm{~g}$ de princípio ativo por 
50kg de semente (REUNIÃO..., 1993) e à inoculação das sementes. A máquina foi lavada com água sanitária diluída e água sob pressão após o preparo de cada tratamento. A calda do fungicida serviu como aderente para o inoculante.

Anteriormente à semeadura, a área foi dessecada com $600 \mathrm{~g} \mathrm{ha}^{-1}$ de i.a. sal isopropilamina de glifosate e, como adjuvante, $1 \%$ de óleo mineral e $2 \%$ de sulfato de amônio. A semeadura foi feita com semeadora de plantio direto, com $35 \mathrm{~cm}$ de espaçamento, a qual também foi desinfestada após cada tratamento, através da retirada das sementes e limpeza com jato de ar. Esta foi realizada em 06/12/94 com o cultivar CEP 12, iniciando-se pela testemunha sem inoculação para evitar contaminação.

No florescimento, avaliaram-se o número e peso de nódulos secos e a massa seca da parte aérea e raízes de 12 plantas por parcela, de uma linha lateral da cultura, na área útil. O teor de nitrogênio foi determinado através da análise de tecido vegetal (TEDESCO et $\boldsymbol{a l}$., 1985), tomando-se por amostra a terceira folha a partir do ápice de 30 plantas por parcela e também foi medida a altura de planta em 5 plantas por parcela. Estas plantas foram escolhidas aleatoriamente, dentro da área útil. Para avaliação do rendimento de grãos, foram colhidas as duas linhas centrais de cada parcela, sendo o resultado expresso em $\mathrm{kg} \mathrm{ha}^{-1}$, a $13 \%$ de umidade.

Os resultados foram avaliados pela análise da variância e as médias dos tratamentos foram comparadas pelo teste de Duncan a 5\% de significância.

\section{RESULTADOS E DISCUSSÃO}

Não houve diferenças entre a testemunha e as doses de inoculante usadas para número de nódulos, peso de nódulos, massa seca da parte aérea e raízes, altura de planta e teor de $\mathrm{N}$ na folha (tabela $1)$.

Quanto ao rendimento de grãos, a testemunha sem inoculação não diferiu estatisticamente dos demais tratamentos, com exceção da dose de $1000 \mathrm{~g}$ de inoculante onde, surpreendentemente, ocorreu redução do rendimento. Nesta dose, há grande probabilidade de perda de inoculante da semente (BRANDÃO JÚNIOR \& HUNGRIA, 1997) o que, entretanto, não explicaria esta redução em relação à testemunha. Este desempenho da testemunha, provavelmente, foi devido à população naturalizada, que neste solo apresentava-se em $10^{3}$ células de Bradyrhizobium/g de solo, que pode ser capaz de garantir bons níveis de nodulação e fixação do $\mathrm{N}_{2}$ (OLIVEIRA \& VIDOR, 1984a).

A aplicação de $200 \mathrm{~kg} \mathrm{ha}^{-1}$ de $\mathrm{N}$ mineral reduziu drasticamente a massa nodular em até $83 \%$ em relação aos demais tratamentos. Entretanto, este tratamento apresentou a maior altura de planta e tendência, apesar de não estatisticamente significativa, de aumento no teor de $\mathrm{N}$ na folha, sem, contudo, resultar em um incremento no rendimento de grãos. A falta de resposta à adubação nitrogenada é um indicativo de que a fixação biológica supriu adequadamente as necessidades da cultura em nitrogênio, para os níveis de produtividade alcançados no experimento.

A resposta da soja à inoculação depende das características de cada solo, sendo que o uso de estirpes de Bradyrhizobium altamente eficientes nem sempre promove aumento no rendimento de grãos, devido à competição por sítios de infecção nodular que normalmente ocorre entre as estirpes utilizadas no inoculante e as estirpes existentes no solo (WEAVER \& FREDERICK, 1974; OLIVEIRA \& VIDOR, 1984b). Acredita-se que as modificações resultantes nas características do solo após alguns anos de plantio direto possam favorecer o desenvolvimento das bactérias do solo, diminuindo assim o efeito da inoculação.

Em trabalho conduzido em laboratório por BRANDÃO JÚNIOR \& HUNGRIA (1997), as doses de 250 e $500 \mathrm{~g}$ de inoculante permitiram maior aderência do inoculante às sementes, com uma queda drástica na dose de $1000 \mathrm{~g}$, quando somente $66 \%$ do inoculante ficou aderido às sementes. Os autores citam que, nas condições atuais, fica claro que doses superiores a $750 \mathrm{~g}$ de inoculante implicam perda de uma grande parte do inoculante adicionado e, pelos problemas causados com o depósito no fundo da semeadora, não se justifica essa recomendação. Nesse mesmo trabalho, num experimento conduzido a campo, em Londrina, PR, os autores não encontraram diferenças no rendimento de grãos e $\mathrm{N}$ total no grão entre as doses de inoculante e a testemunha sem inoculação. Entretanto, no experimento conduzido em Ponta Grossa, PR, em um solo menos fértil, a maior produtividade foi conseguida com a dose de $500 \mathrm{~g}$, servindo de base para a recomendação atual no Estado do Paraná.

REZENDE et al. (1981), trabalhando em solo do cerrado, verificaram que todos os tratamentos inoculados foram superiores à testemunha em termos de produção de grãos, entretanto, não houve efeito significativo das diferentes doses empregadas, de 400 a $2000 \mathrm{~g}$ por $50 \mathrm{~kg}$ de semente. Salienta-se que na região de cerrado as condições para desenvolvimento da população de rizóbio no solo são menos favoráveis que na região Sul, devido à maior acidez do solo, baixa fertilidade e altas temperaturas. 
Tabela 1 - Efeito de doses de inoculantes sobre a nodulação, massa seca da parte aérea e raízes, altura de plantas, teor de N na folha e rendimento de grãos na cultura da soja em plantio direto. FUNDACEP, safra 1994/95.

\begin{tabular}{|c|c|c|c|c|c|c|c|}
\hline Tratamento & $\begin{array}{c}\text { Número de } \\
\text { nódulos } \\
\text { (n⿳/planta) }\end{array}$ & $\begin{array}{c}\text { Peso de } \\
\text { nódulos } \\
\text { (mg/planta) }\end{array}$ & $\begin{array}{c}\text { Massa seca } \\
\text { parte aérea } \\
\text { (g/planta) }\end{array}$ & $\begin{array}{c}\text { Massa seca } \\
\text { de raízes } \\
\text { (g/planta) }\end{array}$ & $\begin{array}{l}\text { Altura de } \\
\text { planta } \\
(\mathrm{cm})\end{array}$ & $\begin{array}{c}\text { Teor de } \mathrm{N} \\
\text { na folha } \\
(\%)\end{array}$ & $\begin{array}{c}\text { Rendimento } \\
\text { de grãos } \\
\left(\mathrm{kg} \mathrm{ha}^{-1}\right)\end{array}$ \\
\hline Testemunha & $39,8 \mathrm{a}^{(1)}$ & $87,8 \mathrm{a}$ & $10,58 \mathrm{~ns}^{(2)}$ & $1,52 \mathrm{~ns}$ & $62,6 \mathrm{~b}$ & $3,79 \mathrm{~ns}$ & $2895 \mathrm{a}$ \\
\hline $250 \mathrm{~g}$ de inoculante & $34,9 \mathrm{ab}$ & $90,1 \mathrm{a}$ & 9,31 & 1,29 & $69,4 \mathrm{~b}$ & 3,64 & $2213 a b$ \\
\hline $500 \mathrm{~g}$ de inoculante & $28,3 \mathrm{abc}$ & $73,7 \mathrm{a}$ & 9,29 & 1,42 & $65,5 \mathrm{~b}$ & 3,91 & $2643 \mathrm{ab}$ \\
\hline $750 \mathrm{~g}$ de inoculante & $29,1 \mathrm{abc}$ & $80,5 \mathrm{a}$ & 8,42 & 1,15 & $66,4 \mathrm{~b}$ & 3,79 & $2239 \mathrm{ab}$ \\
\hline $1000 \mathrm{~g}$ de inoculante & $39,1 \mathrm{ab}$ & $110,3 \mathrm{a}$ & 8,46 & 1,25 & $66,5 \mathrm{~b}$ & 3,82 & $2028 \mathrm{~b}$ \\
\hline $200 \mathrm{~kg}$ de $\mathrm{N} / \mathrm{ha}$ & $16,7 \mathrm{c}$ & $18,4 \mathrm{~b}$ & 13,31 & 1,44 & $78,4 \mathrm{a}$ & 4,31 & $2753 \mathrm{ab}$ \\
\hline $\mathrm{CV}(\%)$ & 33,3 & 37,8 & 27,1 & 17,0 & 6,9 & 9,2 & 18,3 \\
\hline
\end{tabular}

${ }^{(1)}$ Médias seguidas pela mesma letra, na coluna, não diferem estatisticamente pelo teste de Duncan a $5 \%$ de significância.

${ }^{(2)} \mathrm{ns}=$ não significativo pelo teste $\mathrm{F}$ a $5 \%$ de probabilidade.

\section{CONCLUSÃO}

Em área de plantio direto já estabelecido, não há resposta à inoculação das sementes de soja com Bradyrhizobium japonicum.

\section{AGRADECIMENTOS}

Ao funcionário Jorge Antônio de Moraes, demais funcionários, colegas e estagiários que colaboram para realização deste trabalho.

\section{REFERÊNCIAS BIBLIOGRÁFICAS}

BERTONI, J., LOMBARDI NETO, F. Conservação do solo. São Paulo: Icone, 1990. $355 \mathrm{p}$

BRAGAGNOLO, N., MIELNICZUK, J. Cobertura do solo por palha de trigo e seu relacionamento com a temperatura e umidade do solo. Revista Brasileira de Ciência do Solo, Campinas, v. 14, n. 3, p. 369-374, 1990.

BRANDÃO JÚNIOR, O., HUNGRIA, M. Maximização da técnica da inoculação - Efeito da dose de inoculante turfoso. In: EMBRAPA-CNPSo. Resultados de pesquisa da Embrapa Soja. Londrina: EMBRAPA, 1997. p. 56-58.

CAMPOS, B.C. de, REINERT, D.J., NICOLODI, R., et al Estabilidade estrutural de um latossolo vermelho-escuro distrófico após sete anos de rotação de culturas e sistemas de manejo do solo. Revista Brasileira de Ciência do Solo, Campinas, v. 19, n. 1, p. 121-126, 1995.

ELTZ, F.L.F., PEIXOTO, R.T.G., JASTER, F. Efeitos de sistemas de preparo do solo nas propriedades físicas e químicas de um latossolo bruno álico. Revista Brasileira de Ciência do Solo, Campinas, v. 13, n. 2, p. 259-267, 1989.

EMBRAPA-CNPSo/OCEPAR. Recomendações Técnicas para a Cultura da Soja no Paraná 1993/94. Londrina: EMBRAPA, 1993. 128 p.
FREIRE, J.R.J. Fixação do nitrogênio pela simbiose rizóbio/leguminosas. In: CARDOSO, E.J.B.N., TSAI, S.M., NEVES, M.C.P. (coord.). Microbiologia do solo. Campinas: SBCS, 1992. p. 121-140.

OLIVEIRA, L.A., VIDOR, C. Capacidade competitiva de estirpes de Rhizobium japonicum em solos com alta população deste Rhizobium. Revista Brasileira de Ciência do Solo, Campinas, v. 8, n. 1, p. 49-55, 1984a.

OLIVEIRA, L.A., VIDOR, C. Seleção de estirpes de Rhizobium japonicum em soja. II. Capacidade competitiva por sítios de nódulos. Revista Brasileira de Ciência do Solo, Campinas, v. 8 , n. 1 , p. $43-47,1984$ b.

REUNIÃO DE PESQUISA DE SOJA DA REGIÃO SUL, 22. Recomendações Técnicas para a Cultura da Soja no Rio Grande do Sul e Santa Catarina - Safra 1993/94. Santa Rosa: CIENTEC-IPAGRO, 1993. 64 p.

REZENDE, P.M. de, LIMA, L.A.P., JUNQUEIRA NETTO, A. et al. Efeito de doses de inoculantes sobre a nodulação e produção de grãos de soja (Glycine $\max$ (L.) Merril). In: SEMINÁRIO NACIONAL DE PESQUISA DE SOJA, 2., Brasília, 1981. Anais ... Brasília: EMBRAPA, 1981. p. 573582 .

SÁ, J.C.M. Manejo da fertilidade do solo no sistema plantio direto. In.: CNPT-EMBRAPA, FUNDACEP-FECOTRIGO, FUNDAÇÃO ABC. Plantio Direto no Brasil. Passo Fundo: Aldeia Norte, 1993. p. 37-60.

TEDESCO, M., WOLKWEISS, S.J., BOHNEN, H. Análise de solos, plantas e outros materiais. Porto Alegre: UFRGS, 1985. 188 p. Boletim Técnico, 20.

VARGAS, M.A.T., PERES, J.R.R., SUHET, A.R. Adubação nitrogenada, inoculação e épocas de calagem para a soja em um solo sob cerrado. Pesquisa Agropecuária Brasileira, Brasília, v. 17, n. 8, p. 1127-1132, 1982.

WEAVER, R.W. \& FREDERICK, L.R. Effect of inoculum rate on competive nodulation of Glycine $\max$ (L.) Merrill. II. Field studies. Agronomy Journal, Madison, v. 66, p. 233-236, 1974. 